\title{
ISO LWS OBSERVATIONS OF EVOLVED STARS AND NEBULAE
}

\author{
M. J. BARLOW \\ Dept. of Physics \& Astronomy, University College London, \\ Gower Street, London WC1E 6BT, UK
}

\section{Introduction}

This review reports, on behalf of the LWS Consortium ${ }^{1}$, some of the spectroscopic results on evolved stars and nebulae that have been obtained at far-infrared wavelengths by the Infrared Space Observatory (ISO) since its launch in November 1995. The results reported have been obtained mainly using ISO's Long Wavelength Spectrometer (LWS; Clegg et al. 1996), but for some objects complementary spectra have also been obtained with the Short Wavelength Spectrometer (SWS; de Graauw et al. 1996). In this review I present some of the results that have been obtained (a) for oxygenrich cool stars and (b) for carbon stars and carbon-rich post-AGB objects and planetary nebulae. Highlights from the first area include the first detection of the far-infrared rotational lines of water vapour, the dominant coolant in their outflows, and the detailed confirmation of the proposed radiative pump mechanism for circumstellar $\mathrm{OH}$ masers. Highlights from the second area include the detection of a rich $\mathrm{HCN}$ and $\mathrm{CO}$ far-infrared line spectrum from the carbon star IRC $+10^{\circ} 216$, with $\mathrm{HCN}$ found to be the dominant coolant, the detection of very strong shock-excited CO rotational lines from the post-AGB object AFGL 2688, and the first detection of the far-infared rotational lines of $\mathrm{CH}^{+}$(from the the PDR of the carbon-rich planetary nebula NGC 7027), as well as the first detection in emission of the far-IR rotational lines of CH (also from NGC 7027).

\footnotetext{
${ }^{1}$ presented on behalf of members of the ISO LWS Post Main Sequence Specialist Astronomy Group: J.-P. Baluteau, M.J. Barlow, J. Cernicharo, M. Cohen, R.J. Cohen, P. Cox, R.J. Emery, E. Gonzalez-Alfonso, C.Gry, T.Lim, X-W. Liu, A.Omont, D.Péquignot, N-Q.Rieu, B. Swinyard, R.J. Sylvester and Truong-Bach
} 


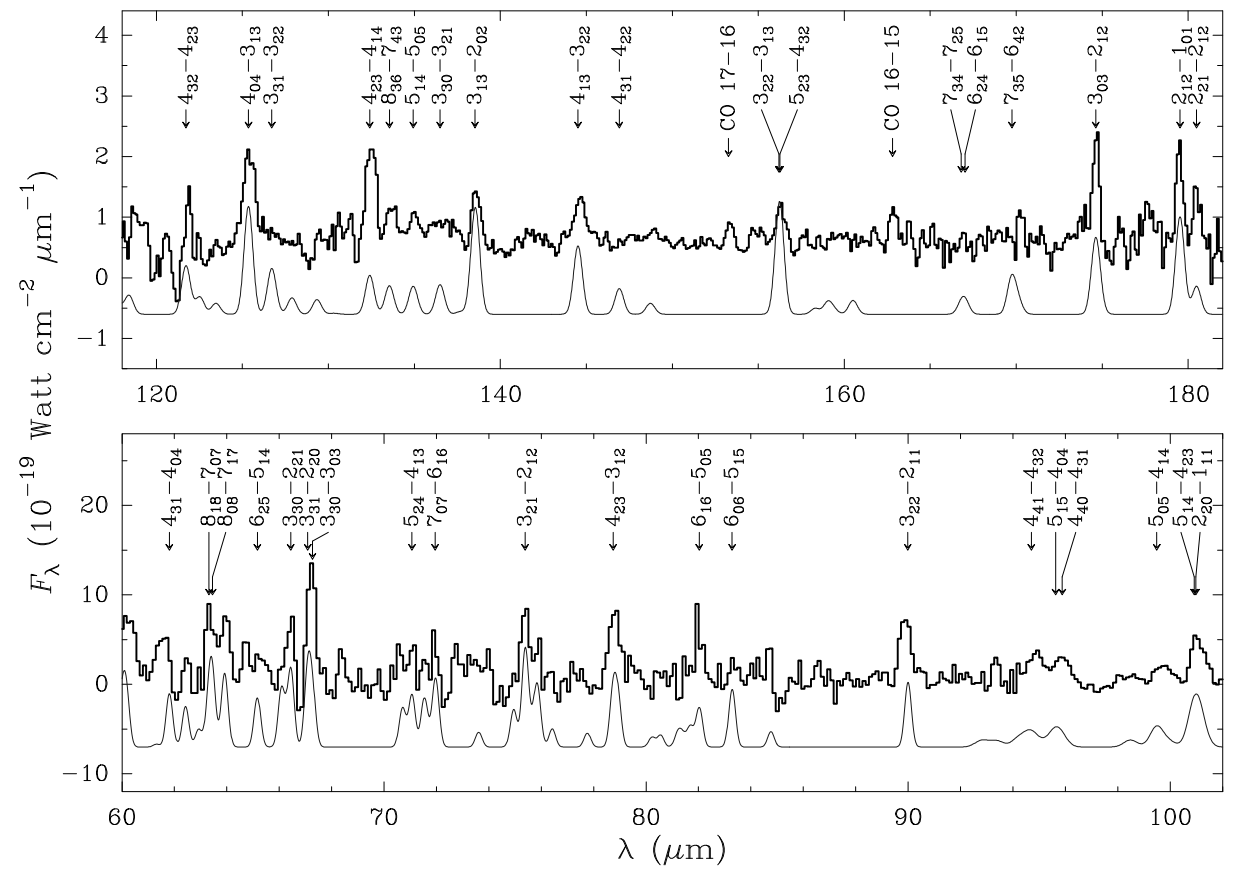

Figure 1. The best-fitting $\mathrm{H}_{2} \mathrm{O}$ line spectrum model is plotted below the continuumsubtracted LWS spectrum of W Hya, with the dominant rotational transitions labelled. From Barlow et al. (1996).

\section{Oxygen-rich cool stars}

\subsection{WATER VAPOUR LINES FROM COOL STARS}

ISO has provided the first extra-solar system detection of the important farinfrared rotational transitions of water molecules. These lines had not been detected previously, even from airborne altitudes, because of the enormous opacity of the Earth's atmosphere in the same lines. Their detection by ISO has confirmed predictions (e.g. Deguchi \& Rieu 1990; Chen \& Neufeld 1995) of the importance of water vapour cooling lines in a variety of astrophysical environments. One of the earliest LWS targets, the oxygen-rich AGB star W Hya, was found to exhibit more than 40 detectable water vapour lines in the 43-180 $\mu \mathrm{m}$ range (Barlow et al. 1996), even at the relatively low LWS grating-mode resolution ( $0.6 \mu \mathrm{m}$ FWHM in first order, longwards of $90 \mu \mathrm{m}$; and $0.3 \mu \mathrm{m}$ in second order, longwards of $43 \mu \mathrm{m})$. Fig. 1 shows portions of the continuum-subtracted LWS spectrum of W Hya, along with their best-fitting model spectrum. Large Velocity Gradient (LVG) Sobolev escape probability models were constructed for the wind of this M8e-M9e semi- 


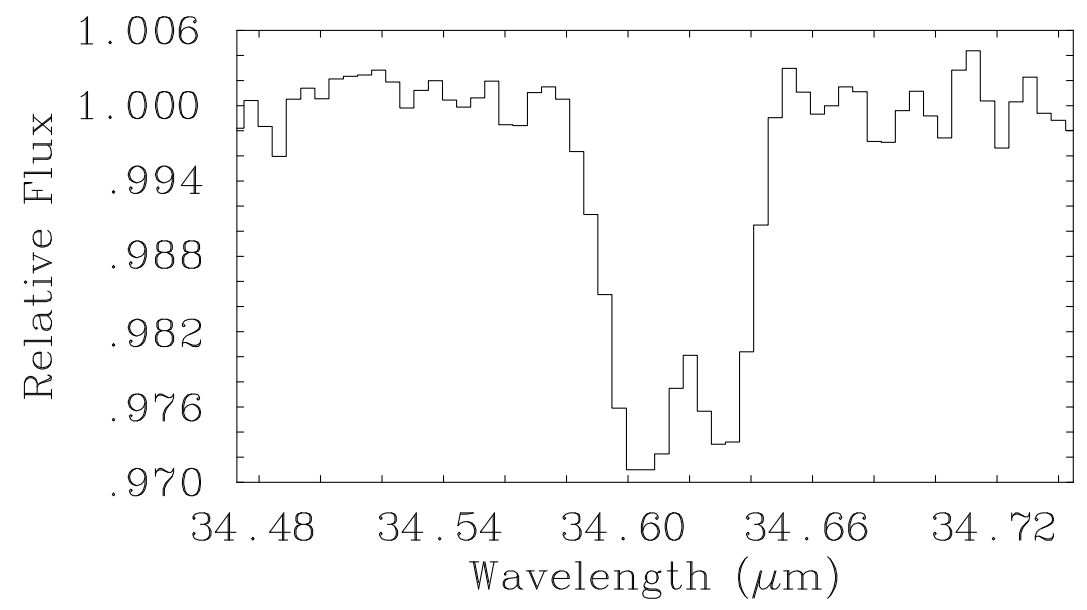

Figure 2. The rectified SWS02 spectrum of IRC $+10^{\circ} 420$ 's $\mathrm{OH} 34.6-\mu \mathrm{m}$ line, which is resolved into its doublet components (rest wavelengths 34.6035 and $34.6294 \mu \mathrm{m}$ ). From Sylvester et al. (1997).

regular variable AGB star in order to try to reproduce the observed water line fluxes. The excitation of $\mathrm{H}_{2} \mathrm{O}$ is dominated by collisions, primarily with $\mathrm{H}_{2}$. The calculated spectrum is found to be sensitive to the adopted mass loss rate and to the $\mathrm{H}_{2} \mathrm{O} / \mathrm{H}_{2}$ abundance ratio, and only weakly dependent on the wind velocity field and temperature structure. All of the observed water lines are found to be very optically thick, explaining the ease with which transitions from the higher pure rotational levels were detected. A first fit with a constant $\mathrm{H}_{2} \mathrm{O} / \mathrm{H}_{2}$ ratio, while matching the fluxes of the low$\mathrm{J}$ transitions (those with excitation energies less than $200 \mathrm{~cm}^{-1}$ ), tended to systematically underestimate the the fluxes of the higher- $\mathrm{J} \mathrm{H}_{2} \mathrm{O}$ lines. These higher-J transitions arise mainly from the innermost regions of the wind, and an improved fit was obtained by adopting a higher $\mathrm{H}_{2} \mathrm{O} / \mathrm{H}_{2}$ number ratio of $8 \times 10^{-4}$ within 4 stellar radii and a lower ratio $\left(3 \times 10^{-4}\right)$ beyond that point. The spectrum predicted by this model is shown in Fig. 1. It corresponds to a mass loss rate of $6 \times 10^{-7} \mathrm{M}_{\odot} \mathrm{yr}^{-1}$ for a distance of $130 \mathrm{pc}$, and has a Goldreich \& Scoville (1976)-type wind temperature distribution (a constant $1300 \mathrm{~K}$ inside $3 \mathrm{R}_{*}$; but decreasing as $\mathrm{r}^{-0.82}$ beyond there).

\subsection{THE OH MASER PUMP MECHANISM FOR IRC $+10^{\mathrm{O}} 420$}

The derived decrease in the abundance of water in the outer parts of the envelope of $\mathrm{W}$ Hya is consistent with photochemical models, which predict that $\mathrm{H}_{2} \mathrm{O}$ should be photodissociated to $\mathrm{OH}$ at larger radii by the 

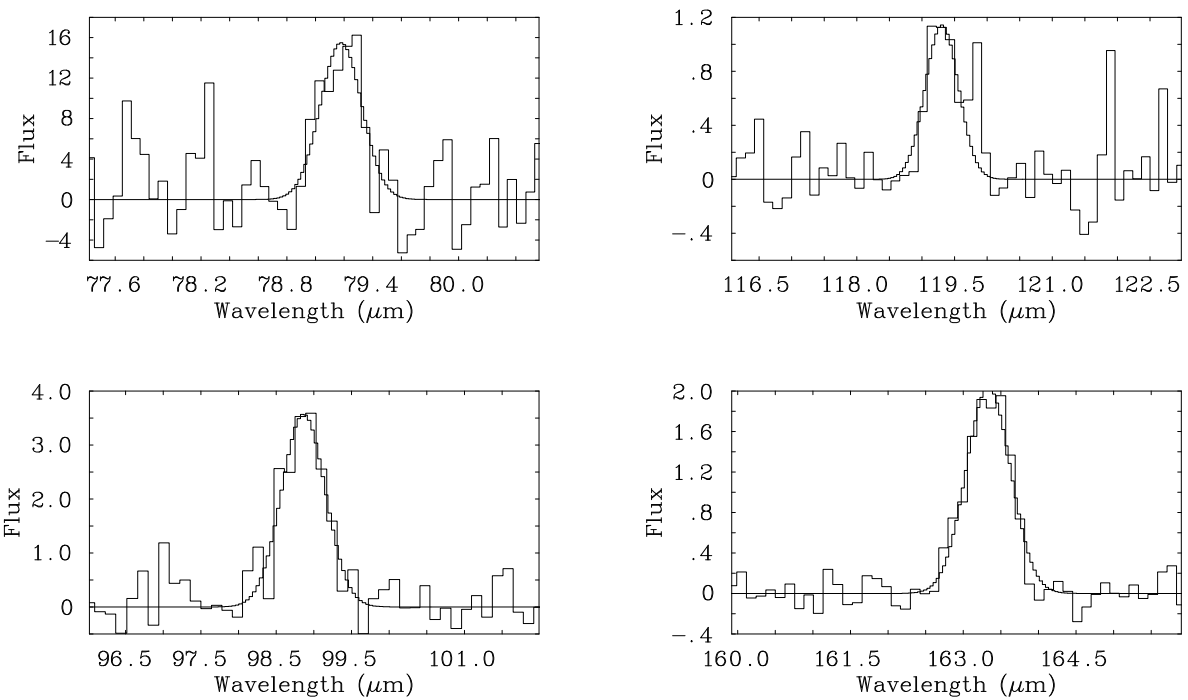

Figure 3. The four detected $\mathrm{OH}$ emission lines in the LWS01 spectrum of IRC $+10^{\circ} 420$. In each plot the underlying continuum has been subtracted. From Sylvester et al. (1997).

interstellar radiation field (e.g. Huggins \& Glassgold 1982). The population inversions which lead to circumstellar hydroxyl $(\mathrm{OH})$ maser emission from cool stars have long been thought to be radiatively pumped (Elitzur, Goldreich \& Scoville 1976; hereafter EGS). However, the proposed pumping mechanism had not been directly observed before now, because the $\mathrm{OH}$ rotational lines involved in this pumping scheme all occur at far-infrared wavelengths, between 30 and $200 \mu \mathrm{m}$, unobservable from the ground but accessible to $I S O$ 's spectrometers.

$\mathrm{OH}$ maser sources exhibit emission from transitions within the $\mathrm{J}=3 / 2$ ${ }^{2} \Pi_{3 / 2}$ ground state. EGS proposed that the upper sublevel of the $1612 \mathrm{MHz}$ transition is inverted by $34.6-\mu \mathrm{m}$ and $53.3-\mu \mathrm{m}$ photons, emitted by dust in the wind, which are absorbed from the ground state up to excited ${ }^{2} \Pi_{1 / 2}$ rotational levels, followed by a cascade back to the ground state, involving the emission of 98.7-, 163.2- and 79.2- $\mu$ m photons. If the transitions to and from the ground state $(34.6,53.3$ and $79.2 \mu \mathrm{m})$ are optically thick, while those between the excited levels are optically thin, this scheme causes an inversion of the $\mathrm{F}=2 / \mathrm{F}=1$ levels of the ground state, leading to $1612 \mathrm{MHz}$ maser action. The scheme also requires that there be sufficient 34.6- and/or $53.3-\mu \mathrm{m}$ flux available: this is provided by continuum emission from dust in the outflow. 
We have observed the infrared $\mathrm{OH}$ pump lines from $\mathrm{IRC}+10^{\circ} 420$. This star is a massive F8Ia hypergiant and a Type II OH maser source, with the $1612 \mathrm{MHz}$ line stronger than the main lines. It is one of the warmest known OH maser stars. Nedoluha \& Bowers (1992) modelled the shell of maseremitting regions as an oblate spheroid with a semi-major axis of $8700 \mathrm{AU}$, with the near side of the equatorial plane tilted $25^{\circ}$ above the line of sight and the outflow velocity increasing from $28 \mathrm{~km} \mathrm{~s}^{-1}$ in the equatorial plane to $63 \mathrm{~km} \mathrm{~s}^{-1}$ along the polar axis. We have obtained SWS grating observations of the $34.6 \mu \mathrm{m}$ pumping line, together with an LWS grating spectrum of the full 43-197 $\mu \mathrm{m}$ region containing the predicted cascade lines that should result from absorption of the dust continuum flux by the $34.6 \mu \mathrm{m}$ line. The $\mathrm{OH}$ 34.6- $\mu \mathrm{m}$ absorption doublet (Fig. 2) and the emission lines in the resulting cascade $(98.7,163.2$ and $79.2 \mu \mathrm{m}$; see Fig. 3) are all clearly detected in the SWS and LWS spectra (Sylvester et al. 1997), confirming that the EGS radiative pumping cycle does indeed operate. Additionally, the $119.3 \mu \mathrm{m} \mathrm{OH}$ doublet is seen in emission (Fig. 3). This ground-state transition may well be collisionally excited: it has the largest cross-section amongst the far-IR $\mathrm{OH}$ transitions for excitation by $\mathrm{H}_{2}$ collisions (Offer \& van Dishoeck 1992).

The observed photon absorption rate in the 34.6 - $\mu \mathrm{m}$ transition is $83 \times 10^{4}$ $\mathrm{m}^{-2} \mathrm{~s}^{-1}$, while the integrated photon flux in the $1612-\mathrm{MHz}$ maser line measured by the Nancay radiotelescope at the time of the ISO observations was 6.2 photons $\mathrm{m}^{-2} \mathrm{~s}^{-1}$. The corresponding pumping efficiency is therefore $7.5 \%$. Since the $1612 \mathrm{MHz}$ maser can also be pumped by radiative absorption by the $53.3 \mu \mathrm{m}$ transition, as well as by radiative absorption and collisional excitation in the $79.2 \mu \mathrm{m}$ line (see Fig. 1 of EGS), a more appropriate estimate for the pumping efficiency comes from ratioing the emission rate in the $1612 \mathrm{MHz}$ maser transition to that in the final downward pumping cascade line at $79.2 \mu \mathrm{m}$. The latter rate is measured to be $(200 \pm 30) \times 10^{4} \mathrm{~m}^{-2} \mathrm{~s}^{-1}$, corresponding to an overall pumping efficiency for the $1612 \mathrm{MHz}$ transition of $3 \%$, in excellent agreement with the predictions of EGS.

\section{Carbon-rich evolved stars and nebulae}

\subsection{IRC $+10^{\mathrm{O}} 216$ AND GL 2688}

The archetypal IR-bright carbon star IRC $+10^{\circ} 216$ was only visible to ISO for a relatively brief period, in the first half of 1996, and was observed with the LWS right at the end of this period. Its far-IR spectrum turned out to be extremely rich in emission lines and these were identified by Cernicharo et al. (1996) with lines of CO and HCN (see the upper spectrum in Fig. 4). The CO pure rotational line spectrum is strongly present, as are the pure 


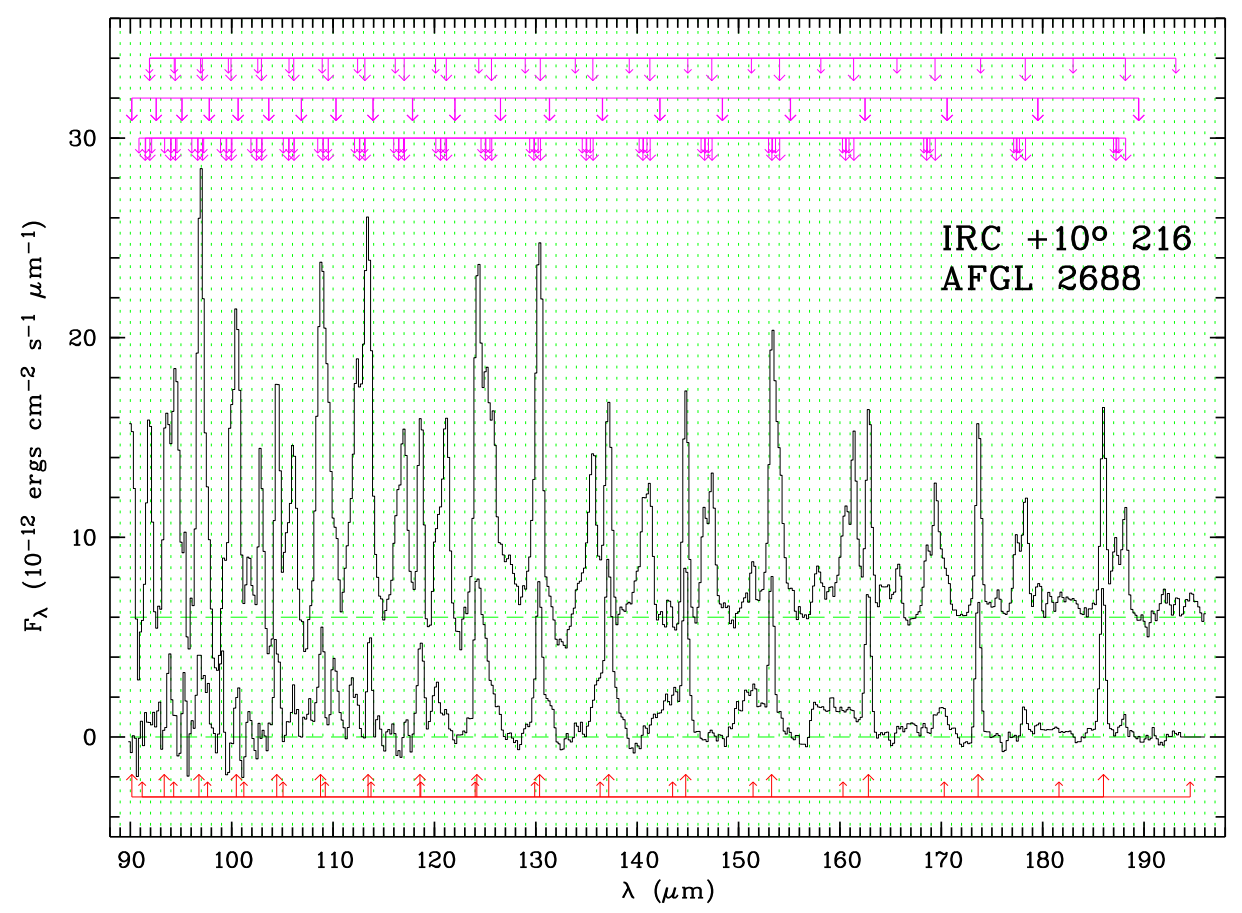

Figure 4. The continuum-subtracted 90-190- $\mu$ m LWS01 spectra of IRC $+10^{\circ} 216$ (upper) and AFGL 2688 (lower). The upward-pointing arrows at the bottom of the plot indicate the positions of pure rotational transitions of ${ }^{12} \mathrm{CO}$ (longer arrows) and ${ }^{13} \mathrm{CO}$ (shorter arrows). The uppermost row of downward-pointing arrows indicates the wavelengths of pure rotational transitions of $\mathrm{H}^{12} \mathrm{CN}$ and $\mathrm{H}^{13} \mathrm{CN}$. The second and third rows of arrows from the top indicate the wavelengths of $\mathrm{HCN}$ rotational transitions from the $\nu_{2}=1,2$ and $\nu_{1,3}=1$ bending vibrational modes.

rotational lines of $\mathrm{HCN}$, but in addition a forest of strong, blended emission features is present. Cernicharo et al. (1996) identified these new features with rotational transitions from bending vibrational excited modes of $\mathrm{HCN}$. From modelling of the wind they derived a HCN/CO number ratio of 0.1. Although HCN was derived to be the less abundant of the two molecules, its larger dipole moment leads to it being the dominant coolant in the wind $\left(0.44 \mathrm{~L}_{\odot}\right.$ in the $\mathrm{HCN}$ lines versus $0.28 \mathrm{~L}_{\odot}$ in the $\mathrm{CO}$ lines $)$.

Deep LWS grating spectra (16000 secs) of the carbon-rich post-AGB object AFGL 2688 (the 'Cygnus Egg'; F5I central star) were acquired during the early Performance Verification phase of ISO (Cox et al. 1996). The 90$190 \mu \mathrm{m}$ continuum-subtracted spectrum of AFGL 2688 is shown in Fig. 4 (lower spectrum). It is dominated by the pure rotational line spectrum of CO (three high-J CO lines had earlier been detected with the KAO by Justtanont et al. 1997). At the longest wavelengths, several high-J rotational 
lines of $\mathrm{HCN}$ were detected, but they are significantly weaker than the $\mathrm{CO}$ lines (by an order of magnitude compared to the case of IRC $+10^{\circ} 216$; upper plot in Fig. 4). The [O I] 63 and $146-\mu \mathrm{m}$ lines and the [C II] $158-\mu \mathrm{m}$ line were not detected, although these lines become the dominant lines from the neutral zone once photodissociation of the molecular envelope begins during the subsequent PN phase. A number of other, relatively strong and broad, emission features are apparent in the spectrum of AFGL 2688 plotted in Fig. 4 - these features do not appear to be due to HCN and remain to be identified.

Cox et al. considered two models for the excitation of the CO lines. One was a standard AGB-wind type model, with a mass loss rate of $1.7 \times 10^{-4}$ $\mathrm{M}_{\odot} \mathrm{yr}^{-1}$, a $\mathrm{CO} / \mathrm{H}_{2}$ ratio of $6 \times 10^{-4}$, and an $\mathrm{r}^{-0.65}$ temperature distribution with a starting temperature of $235 \mathrm{~K}$ at a radius $\mathrm{r}$ of $2 \times 10^{16} \mathrm{~cm}$, which corresponds to the dust temperature at that radius previously derived from mid-infrared data. Although capable of matching the relative intensities of the CO lines, this model was unable to match the observed CO line flux levels. Since mm-wave and near-IR observations have provided evidence for high-velocity shock-heated molecular gas $\left(\mathrm{CO}\right.$ and $\left.\mathrm{H}_{2}\right)$ in AFGL 2688, Cox et al. therefore constructed a shock-excitation model for the $\mathrm{CO}$ emission. The LWS data were found to be well-fit by $400 \mathrm{~K}$ molecular gas with a total mass of $0.005 \mathrm{M}_{\odot}$.

\subsection{THE CARBON-RICH PLANETARY NEBULA NGC 7027}

The carbon-rich planetary nebula NGC 7027 lived up to its reputation of always producing suprises when it is observed with new infrared instrumentation. Liu et al. (1996) presented a deep (14500 sec) LWS grating spectrum of NGC 7027 acquired during ISO's PV-phase. In addition to the expected ionic fine structure emission lines from the ionized zone, the spectrum showed strong fine structure lines of [O I] and [C II], arising from about $0.15 \mathrm{M}_{\odot}$ of dissociated molecular gas in the photodissociation region (PDR) outside the ionized region; and a rich $\mathrm{CO}$ rotational line spectrum that could be well fitted by emission from $1000 \mathrm{~K}$ (PDR) gas with a $\mathrm{H}_{2}$ density of $10^{5} \mathrm{~cm}^{-3}$. The major suprise though was the detection of emission in the ground-state $119.3-\mu \mathrm{m} \mathrm{OH}$ doublet, with the tentative detection of emission in the ground-state $\mathrm{OH} 79.2$ and $84.5-\mu \mathrm{m}$ lines, and the apparent detection of strong emission in the ground-state rotational line of $\mathrm{o}-\mathrm{H}_{2} \mathrm{O}$ at $179.53 \mu \mathrm{m}$. The $\mathrm{OH}$ and $\mathrm{H}_{2} \mathrm{O}$ molecules were interpreted by Liu et al. (1996) as having formed in the PDR of this carbon-rich nebula following the dissociation of $\mathrm{CO}$ into atomic $\mathrm{C}$ and $\mathrm{O}$ and the reaction of the latter with $\mathrm{H}_{2}$ molecules to yield (succesively) $\mathrm{OH}$ and $\mathrm{H}_{2} \mathrm{O}$, with the $1000 \mathrm{~K}$ temperature of the PDR enabling the activation energies for these reactions 

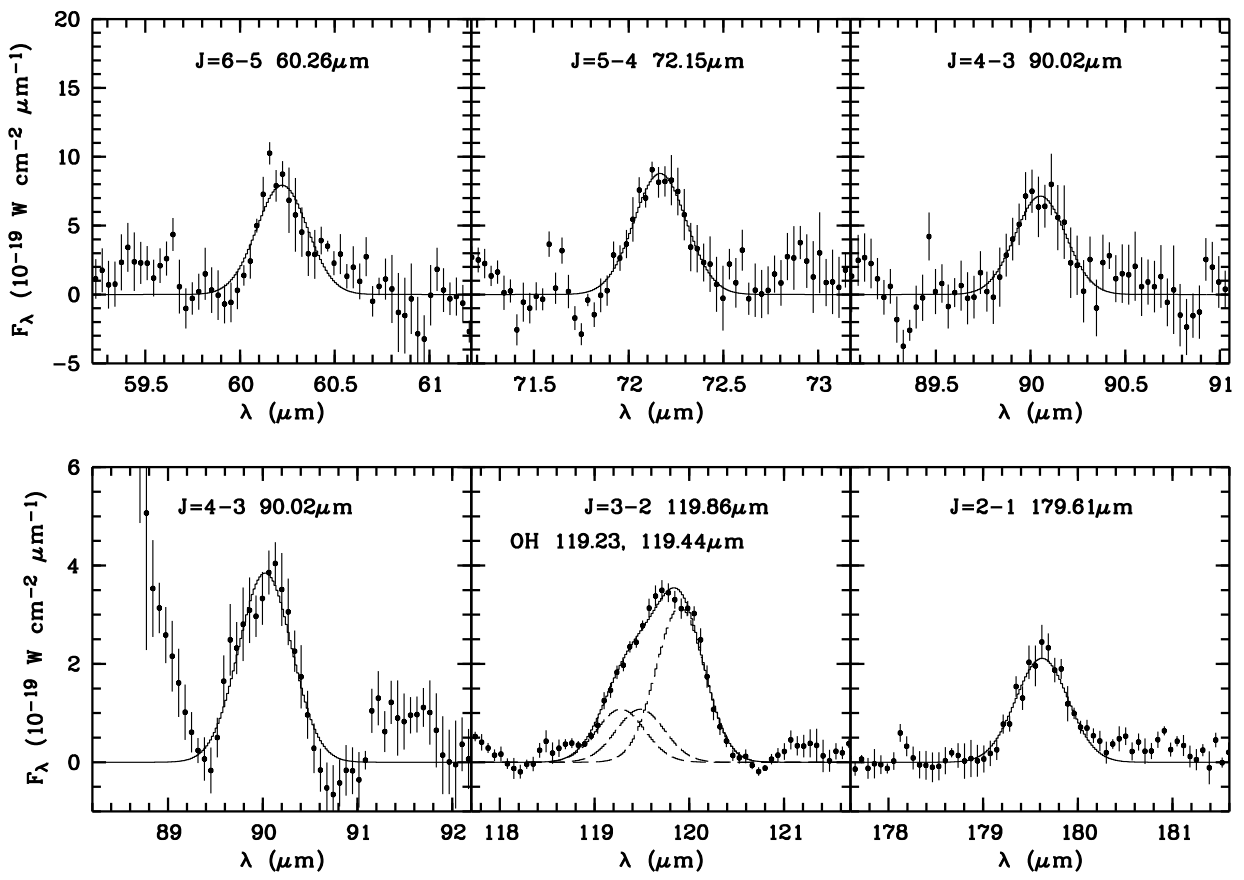

Figure 5. $\mathrm{CH}^{+}$rotational lines observed in NGC7027. The solid lines are gaussian line profile fits to the observed features (for the case of blends, the contributions from individual components are shown as dashed lines). The $\mathrm{J}=2-1$ line of $\mathrm{CH}^{+}$at $179.62 \mu \mathrm{m}$ had been previously assigned to $\mathrm{H}_{2} \mathrm{O}$. For the $\mathrm{J}=4-3$ line $\mathrm{CH}^{+}$line, spectra from two different detectors - LW1 (bottom panel) - where the sharp rise at short wavelengths is due to the strong [O III] $88.36-\mu \mathrm{m}$ line - and SW5 (upper panel) - are shown. The $\mathrm{J}=4-3$ line is blended with the $\mathrm{J}=29-28$ line of $\mathrm{CO}$ at $90.16 \mu \mathrm{m}$. From Cernicharo et al. (1997).

to be overcome.

The identification of the line at $179.6 \mu \mathrm{m}$ with o- $\mathrm{H}_{2} \mathrm{O}$ was overturned by Cernicharo et al. (1997), who showed that it was due to the $\mathrm{J}=2-1$ rotational line of $\mathrm{CH}^{+}$at $179.62 \mu \mathrm{m}$. This identification was supported by the simultaneous identification of the $\mathrm{J}=3-2,4-3,5-4$ and $6-5$ lines of $\mathrm{CH}^{+}$in the LWS spectrum, whose $\mathrm{S} / \mathrm{N}$ had been significantly increased by the availability of a new calibration and the addition of several more routine-phase observations, yielding a total integration time of 24000 secs. The newly identified $\mathrm{CH}^{+}$lines are illustrated in Fig. 5. The $\mathrm{J}=3-2$ line of $\mathrm{CH}^{+}$at $119.9 \mu \mathrm{m}$ (middle-bottom panel of Fig. 5) is clearly blended with the $119.3-\mu \mathrm{m}$ doublet of $\mathrm{OH}$. In addition, the new spectrum provided clear detections of the $\mathrm{OH} 79.2$ and $84.5-\mu \mathrm{m}$ lines. The presence of $\mathrm{H}_{2} \mathrm{O}$ must be considered doubtful at the moment, although three lines of $\mathrm{o}-\mathrm{H}_{2} \mathrm{O}$, at 174.6, 108.05 and 75.4- $\mu \mathrm{m}$ are tentatively identified. The formation of $\mathrm{CH}^{+}$, 
via the reaction $\mathrm{C}^{+}+\mathrm{H}_{2} \rightarrow \mathrm{CH}^{+}+\mathrm{H}$, requires an activation energy of $4000 \mathrm{~K}$, which can be supplied by the warm PDR.

The formation of $\mathrm{CH}^{+}$and $\mathrm{OH}$ in the PDR around NGC 7027 had been predicted by Black (1978), who also predicted that $\mathrm{HeH}^{+}$might be formed in sufficient quantities to be detectable. The $\mathrm{HeH}^{+}$molecule is of interest as it is one of the few molecules predicted to be capable of forming in the early post-recombination phase of the Universe. It has never been detected astrophysically, but NGC 7027 has been considered to be one of the most promising sites for its detection, due to the high density and high degree of ionization of the nebula $\left(\mathrm{HeH}^{+}\right.$is, unusually, predicted to be formed within the ionized zone, via the reaction $\mathrm{He}^{+}+\mathrm{H} \rightarrow \mathrm{HeH}^{+}+\gamma$; see Cecchi-Pestellini \& Dalgarno 1993, hereafter CD93). $\mathrm{HeH}^{+}$has its $\mathrm{J}=1-0$ fundamental pure rotational line at $149.14 \mu \mathrm{m}$. Liu et al. (1997) analysed the deep LWS spectrum of NGC 7027 in a search for this line. They detected an emission feature at about the correct wavelength and with a flux in agreement with that predicted by $\mathrm{CD} 93$. However, the $\mathrm{CH}$ radical has its $\mathrm{J}=3 / 2-1 / 2$ fundamental pure rotational doublet at 149.09 and $149.39 \mu \mathrm{m}$. The presence in the NGC 7027 spectrum of the $\mathrm{CH} \mathrm{J}=5 / 2-3 / 2$ doublet at 180.48 and $180.93 \mu \mathrm{m}$, with a flux consistent with the observed feature at $149.18 \mu \mathrm{m}$ being entirely due to $\mathrm{CH}$, led Liu et al. (1997) to conclude that the $\mathrm{HeH}^{+} \mathrm{J}=2-1$ line was not detected and to assign an upper limit to its flux that was three times lower than predicted by CD93. The $\mathrm{CH}$ line identifications represent the first detection of the pure rotational emission line spectrum of $\mathrm{CH}$ from an astrophysical object. Liu et al. (1997) derived a $\mathrm{CH} / \mathrm{CO}$ abundance ratio of $\sim 0.06$ and a $\mathrm{CH} / \mathrm{CH}^{+}$ratio of $\sim 0.2$.

\section{References}

Barlow M. J., et al., 1996, A\&A, 315, L241

Black J. H., 1978, ApJ, 222, 125

Cecchi-Pestellini C. \& Dalgarno A., 1993, ApJ, 413, 611 (CD93)

Cernicharo J., et al., 1996, A\&A, 315, L201

Cernicharo J., et al., 1997, ApJL, 483, L65

Chen W., Neufeld D.A., 1995, ApJL, 453, L99

Clegg P. E., et al., 1996, A\&A, 315, L38

Cox P., et al., 1996, A\&A, 315, L265

de Graauw Th., et al., 1996, A\&A, 315, L49

Deguchi S., Rieu N-Q., 1990, ApJL, 360, L27

Elitzur M., Goldreich P., Scoville N., 1976, ApJ, 205, 384 (EGS)

Huggins P., Glassgold A. E., 1982, AJ, 87, 1828

Goldreich P., Scoville N., 1976, ApJ, 205, 144

Justtanont K., Tielens A. G. G. M., Skinner C. J., Haas M. R., 1997, ApJ, 476, 319

Liu X.-W., et al., 1996, A\&A, 315, L257

Liu X.-W., et al., 1997, MNRAS, 290, L71

Nedoluha G. E., Bowers P. F., 1992, ApJ, 392, 249

Offer A. R., van Dishoeck E. F., 1992, MNRAS, 257, 377

Sylvester R. J., et al., 1997, MNRAS, in press 